\title{
VIGILANCIA INTENSIFICADA SOBRE EL DENGUE Y LOS PRIMEROS CASOS DE DENGUE HERMORRAGICO CONFIRMADOS EN COLOMBIA DURANTE EL PRIMER SEMESTRE DE 1990
}

\author{
CECILIA SAAD ACOSTA*, MANCEL MARTINEZ DURAN*, FERNANDO DE LA HOZ*, JOSE PARRA \\ FONSECA* ${ }^{*}$, FABIO RIVAS MUÑOZ**, JORGE BOSHELL SAMPER***, LAURA SOFIA DE CALDERON***, \\ GLADYS MARQUEZ***
}

\begin{abstract}
Se presentan los resultados arrojados por la Vigilancia Activa, ejercida por el Sistema Nacional de Salud, sobre Dengue Clásico (DC), Dengue Clásico con Manifestaciones Hemorrágicas (DC/MH), Dengue Hemorrágico $(\mathrm{DH})$ y Dengue Hemorrágico con Síndrome de Shock (DH/ SS), durante el primer semestre de 1990.
\end{abstract}

De las 3.098 muestras recibidas se confirmaron 68 casos de DC $(18 \%)$, 121 de ellos presentaron manifestaciones hemorrágicas $(21 \%)$ y 35 llenaron los requisitos de la OMS para ser considerados como DH (6\%). De estos últimos, 9 presentaron shock (26\%), de los cuales uno fue fatal (3\%).

Las regiones con mayor incidencia de DC fueron: Arauca y Casanare, y para DH: Arauca, Magdalena y Norte de Santander.

Por ser la primera vez que en Colombia se confirma la presencia de $\mathrm{DH}$, se describen las principales características clínico-epidemiológicas observadas en los primeros casos presentados.

\section{INTRODUCCION}

Debido a la aparición de un brote de DH en Venezuela, en Colombia se activó la Vigilancia Intensificada sobre el DC y la búsqueda de casos de DH, valiéndose de todos los componentes de la Vigilancia Activa, a saber: vigilancia clínica, serológica, virológica, entomológica y epidemiológica.

Hasta enero de 1990 no se había podido confirmar ningún caso de DH en el país, pero gracias a la Vigilancia Intensificada y a la participación de los servicios de salud, fue posible hacerlo durante el primer semestre del presente año; sin embargo, desde 1970 se ha tenido circulación de los cuatro serotipos del virus, así como la presencia de características epidémicas similares a la de países latinoamericanos que han padecido epidemias de DH/SS $(7,8)$.

\section{MATERIALES Y METODOS}

Durante la segunda semana de enero se envió a los servicios de salud del país, una guía sobre Vigilancia y Control del DC y del DH en Colombia; además se impartieron intrucciones para el diagnóstico de casos sospechosos de DC y de DH, notificación de casos, toma y remisión de muestras de sueros al Laboratorio de Virología del Instituto Nacional de Salud (INS) (1).

Con base en las instrucciones impartidas, los servicios de salud iniciaron la búsqueda activa de pacientes febriles con manifestaciones de DC y de DH. A los

* Grupo de Epidemiología, INS

** División Investigaciones Especiales, INS

***Grupo de Virología, INS. 
pacientes detectados se les tomó una muestra de suero, la cual fue remitida al INS. En el Laboratorio de Virología todas las muestras fueron procesadas para pruebas serológicas mediante la técnica de Inhibición de la Hemaglutinación (HI) e IgM-ELISA; además, aquellas que fueron tomadas a pacientes en la fase aguda de la enfermedad, se procesaron para intentos de aislamiento en cultivos celulares e inoculación de mosquitos.

Los datos clínico-epidemiológico de los casos se tomaron de las notas de remisión que acompañaban a cada muestra de suero.

Para el diagnóstico se tomaron en cuenta los criterios recomendados por la OMS (5), y los de SCOOTT para determinar el tipo de respuesta serológica, quien define como respuesta primaria: aquellas que en el segundo suero presentan seroconversiones con títulos que no sobrepasan de 1:640 (9).

Los resultados se ordenaron según edad y sexo de los pacientes, de esta manera se obtuvieron porcentajes de positividad para cada uno de estos grupos.

Se calcularon tasas de ataque para cada servicio de salud, teniendo en cuenta el número de casos confirmados por laboratorio y el número de habitantes en las poblaciones donde se obtuvieron las muestras. (2)

Para saber si habían diferencias significativas en los títulos HI de la primera muestra entre personas con dengue y sin él: comparamos los títulos de anticuerpos $\mathrm{HI}$ alcanzados en la primera muestra de 150 pacientes, cuyo segundo suero mostró seroconversión, contra los títulos de 232 pacientes cuyo segundo suero no la mostró. Para la comparación se tuvo en cuenta únicamente el título más alto alcanzado por cualquiera de los anticuerpos HI (FA, D1, D2, D3 y D4).

Utilizamos la transformación logarítmica de los títulos HI para hallar el promedio geométrico de cada grupo, y luego comparamos entre sí los dos promedios, utilizando para esto la prueba t (3).

Utilizamos la prueba z para comparar proporciones a un nivel de significancia de 0.05 (6).

En la tabla 1 observamos el total de muestras recibidas durante el primer trimestre de 1990. Este número ascendió a 3.098, de las cuales el 18\% correspodieron a personas infectadas con el virus del dengue.

$54 \%$ de las muestras recibidas eran de pacientes varones y $46 \%$ pertenecían a mujeres. $\mathrm{p}<0.05$.

El 58\% de los casos confirmados pertencían al grupo entre 15 y 44 años, el $16 \%$ al grupo entre 5 y 14 años, el $14 \%$ a los mayores de 45 años y el $12 \%$ a los menores de 5 años.

La tabla 2 muestra la distribución de los diagnósticos de DC, DC/MH y DH por edad y sexo.

El 94\% de todos los casos de dengue, correspondieron a la categoria de DC, $16 \%$ de éstos tuvieron manifestaciones hemorrágicas pero no llenaron los requisitos para ser considerados como DH y se catalogaron como DC/MH; finalmente, el $6 \%$ de los pacientes con dengue llenaron los requerimientos para ser considerados como DH.

La figura 1 ilustra el porcentaje de positividad por grupos de edad y sexo.

TABLA 1

DISTRIBUCION POR EDAD Y SEXO DE LOS CASOS SOSPECHOSOS DE DENGUE Y DE LOS CONFIRMADOS POR SEROLOGIA (HI)

\begin{tabular}{cccccc}
\hline $\begin{array}{c}\text { Edad } \\
\text { (años) }\end{array}$ & Sexo & $\begin{array}{c}\text { Sospe- } \\
\text { chosos }\end{array}$ & & \multicolumn{2}{c}{$\begin{array}{c}\text { Confir- \% de Po- } \\
\text { mados sitividad }\end{array}$} \\
\hline $0-4$ & $\mathrm{M}$ & 275 & & 33 & 12 \\
& $\mathrm{~F}$ & 258 & & 33 & 13 \\
$5-14$ & $\mathrm{M}$ & 335 & & 43 & 13 \\
& $\mathrm{~F}$ & 282 & & 46 & 16 \\
& & & $\mathrm{p} 0.05$ & & \\
$15-44$ & $\mathrm{M}$ & 837 & & 166 & 20 \\
& $\mathrm{~F}$ & 663 & & 166 & 25 \\
& & & $\mathrm{p} 0.05$ & & \\
$>45$ & $\mathrm{M}$ & 216 & & 31 & 14 \\
& $\mathrm{~F}$ & 232 & & 50 & 21 \\
Subtotal & $\mathrm{M}$ & 1.663 & & 273 & 16 \\
& $\mathrm{~F}$ & 1.435 & & 295 & 10 \\
& & & $\mathrm{p} 0.05$ & & \\
\hline Total & & 3.098 & & 568 & 18 \\
\hline
\end{tabular}


VIGILANCIA INTENSIFICADA SOBRE EL DENGUE Y LOS PRIMEROS CASOS DE DENGUE HEMORRAGICO CONFIRMADOS EN COLOMBIA .......

TABLA 2

DISTRIBUCION POR EDADY SEXO DE LOS CASOS DE DC, DC/MH Y DH

\begin{tabular}{ccrrrr}
\hline $\begin{array}{c}\text { Edad } \\
\text { (años) }\end{array}$ & Sexo & DC & DC/MH & DH & Total \\
\hline $0-4$ & M & 25 & 4 & 4 & 33 \\
& $\mathrm{~F}$ & 25 & 5 & 3 & 33 \\
$5-14$ & $\mathrm{M}$ & 32 & 8 & 3 & 43 \\
& $\mathrm{~F}$ & 28 & 13 & 5 & 46 \\
$15-44$ & $\mathrm{M}$ & 131 & 25 & 10 & 166 \\
& $\mathrm{~F}$ & 136 & 23 & 7 & 166 \\
$>45$ & $\mathrm{M}$ & 24 & 4 & 3 & 31 \\
& $\mathrm{~F}$ & 46 & 4 & 0 & 50 \\
Subtotal & $\mathrm{M}$ & 212 & 41 & 20 & 273 \\
& $\mathrm{~F}$ & 235 & 45 & 15 & 295 \\
\hline Total & & 447 & 86 & 35 & 568 \\
\hline
\end{tabular}

POSITIVIDAD PARA DENGUE

DISTRIBUCION POR EDAD Y SEXO

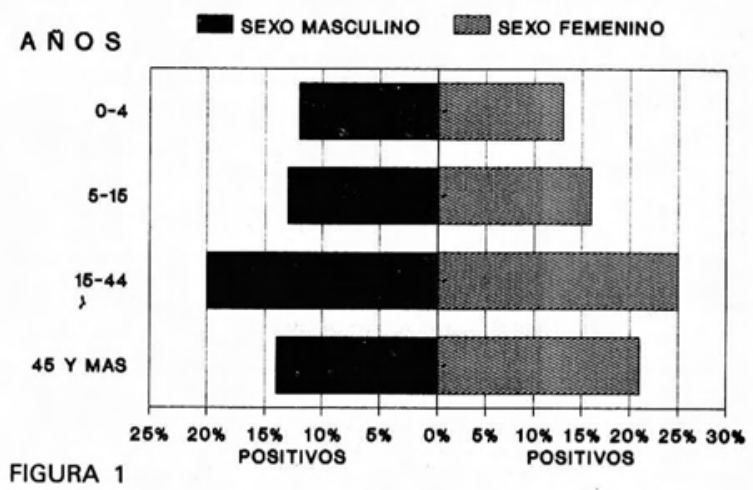

Se observa una tendencia entre las mujeres a tener mayor porcentaje de positividad; sin embargo, sólo en el grupo entre 15-44 años esta diferencia es realmente significativa, estando al borde de la significancia estadística la diferencia por sexos en el grupo mayor de 45 años.

La tabla 3 evidencia que el porcentaje de positividad para DH es similar para todos los grupos de edad. Tampoco hay diferencia entre los grupos de edad en el porcentaje de positividad para DC/MH.
$20 \%$ de los casos de DH se registraron durante la séptima semana epidemiológica de 1990 y el $17 \%$ de los casos de DC se diagnósticaron durante la décima semana epidemiológica.

La figura 2 muestra el porcentaje total de casos de dengue diagnosticados durante cada una de las semanas epidemiológicas estudiadas.

La tabla 4 muestra las tasas de incidencia de DC por regiones del país. Las más afectadas fueron: Arauca, Casanare, Córdoba, Guaviare, Magdalena, Meta y Norte de Santander. Estas conforman el grupo de mayor riesgo para DC.

Con riesgo moderado aparecen: Amazonas, Cundinamarca, Guainía, Guajira, Huila, Nariño y Tolima.

TABLA 3

\section{PORCENTAJES DE POSITIVIDAD PARA DH POR GRUPO DE EDAD EN EL TOTAL DE MUESTRAS RECIBIDAS}

\begin{tabular}{lcrc}
\hline $\begin{array}{l}\text { EDAD } \\
\text { (años) }\end{array}$ & Total de & DH & $\begin{array}{c}\% \text { de Posi- } \\
\text { tividad }\end{array}$ \\
\hline $0-4$ & 533 & 7 & 1 \\
$5-14$ & 617 & 8 & 1 \\
$15-44$ & 1.500 & 17 & 1 \\
$>45$ & 448 & 3 & 0.7 \\
\hline Total & 3.098 & 35 & 1 \\
& & 0.05 & \\
\hline
\end{tabular}

DIAGNOSTICOS DE DENGUE PORCENTAJE POR SEMANAS EPIDEMIOLOGICAS

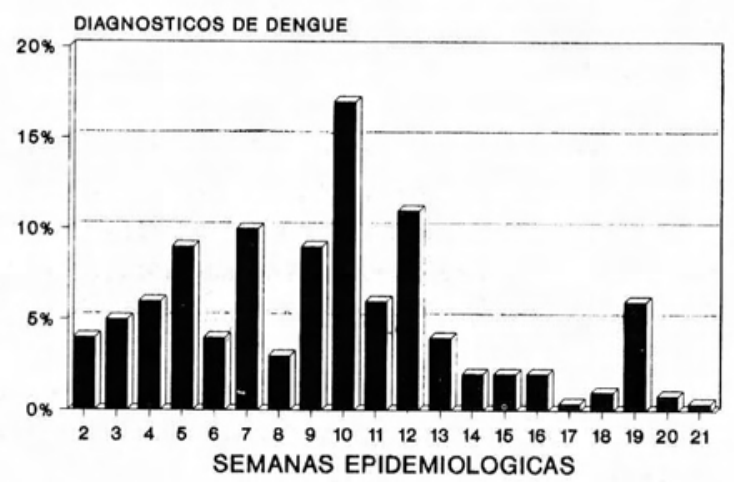

FIGURA 2 


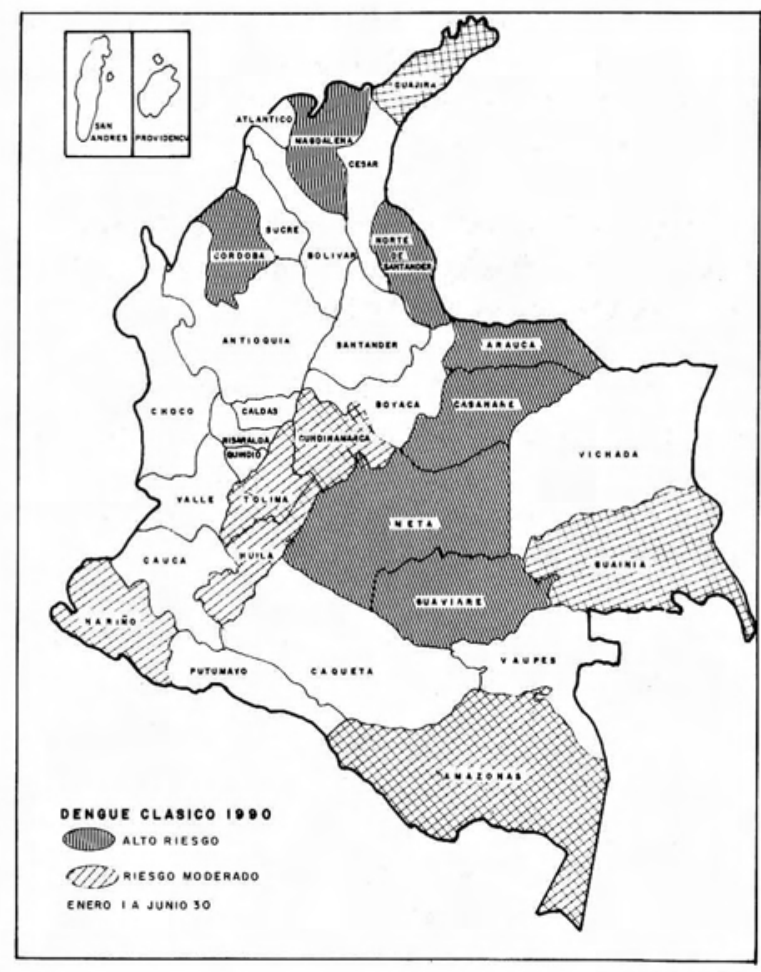

El Laboratorio de Virología aisló el serotipo 4 en muestras de suero procedentes de Cúcuta (Norte de Santander), Fusagasugá (Cundinamarca), Fonseca (Guajira), Pereira (Risaralda), Puerto Berrío (Antioquia), Tumaco (Nariño) y Yopal (Casanare). Se aisló también el Serotipo 1 en Orocué (Casanare) y Pereira (Risaralda).

\section{La figura 3 ilustra la incidencia del DH por regiones:}

Las regiones con mayor incidencia de $\mathrm{DH}$ fueron: Arauca y Magdalena, consideradas de alto riesgo para esta patología.

Con riesgo moderado aparecen: Antioquia, Córdoba, Cundinamarca, Huila, Norte de Santander, Risaralda, Santander, Tolima y Valle. Las regiones que no resportaron casos de $\mathrm{DH}$ las consideramos de bajo riesgo para la ocurrencia de esta patología.

El promedio geométrico de títulos HI, en la primera muestra de personas que no mostraron seroconversión, posteriormente fue de 1:20 con una desviación estándar de 1:10.

TABLA 4

PROPORCION DE INCIDENCIA DEL DENGUE CLASICO POR REGIONES

\begin{tabular}{lrl}
\hline Región & Incidencia por $\mathbf{1 0 0 0}$ habitantes \\
\hline Casanare & 190 & \\
Arauca & 61 & \\
Meta & 26 & \\
Guaviare & 25 & \\
Norte de Santander & 22 & \\
Magdalena & 21 & \\
Córdoba & 19 & \\
& & \\
Guainía & 10 & \\
Cundinamarca & 9 & \\
Huila & 8 & \\
Nariño & 8 & \\
Tolima & 7 & \\
Guajira & 7 & \\
Amazonas & 6 & \\
& & \\
Chocó & 0.05 \\
Caquetá & 4 & \\
Boyacá & 4 & \\
Cesar & 3 & \\
Cauca & 3 & \\
Atlántico & 2 & \\
Risaraldá & 2 & \\
Antioquia* & 2 & \\
Caldas & 2 & \\
Santander & 2 & \\
Valle*- & 1 \\
Bolívar & 0.2 \\
Vichada & 0 \\
Putumayo & 0
\end{tabular}

*Estas Seccionales realizan sus propias pruebas $\mathrm{HI}$ por lo que las muestras envidas al INS correspondian sólo a sospechosos de FHF.

DENGUE HEMORRAGICO
INCIDENCIA POR SECCIONALES

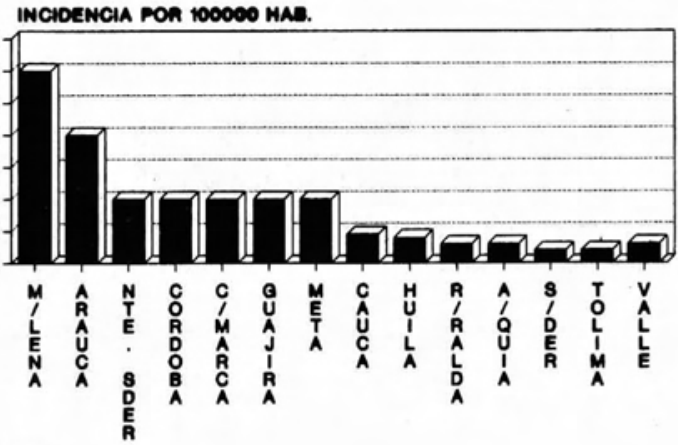

INGIOENCIA PON 100000 HAR.

FIGURA 3 
El promedio de los títulos de la primera muestra, en personas que si seroconvirtieron posteriormente, fue de 1:80, con una desviación de 1:20.

La diferencia de títulos entre los dos grupos de personas, fue significativa. $\mathrm{p}<0.05$.

En los primeros tres días de enfermedad el promedio geométrico de ambas muestras fue similar, 1:40, entre el cuarto y séptimo día; el promedio entre los que seroconvirtieron fue de 1:80 y de 1:20 para los que no seroconvirtieron; luego entre el octavo y decimosegundo día de enfermedad, el promedio entre los que seroconvirtieron fue de 1:80 y de 1:10 para los que no seroconvirtieron. Esta diferencia fue aún más significante que la hallada en la comparación global. p $<0.01$.

La tabla 5 muestra la distribución de casos de DH por edad, sexo y severidad del cuadro clínico, 26 de los cuales fue leve y 9 severos, con una letalidad del $3 \%$ (falleció con un caso entre los severos).

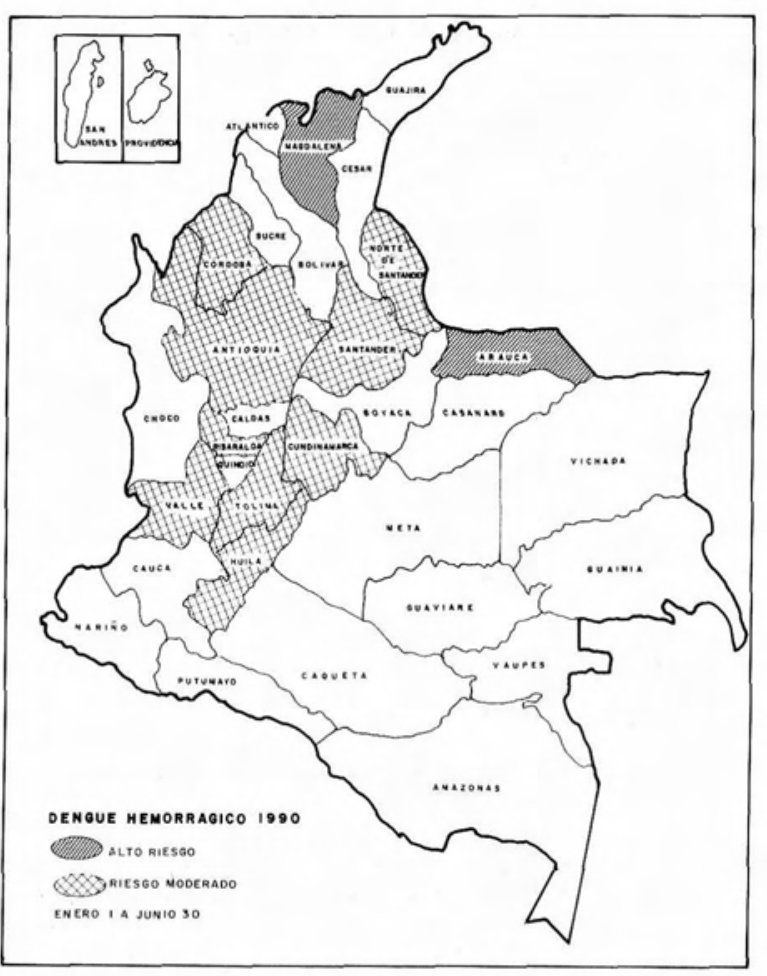

TABLA 5

DISTRIBUCION POR EDAD, SEXO Y SEVERIDAD DEL DH

\begin{tabular}{|c|c|c|c|c|}
\hline \multirow{2}{*}{$\begin{array}{l}\text { Edad } \\
\text { (años) }\end{array}$} & \multirow[t]{2}{*}{ Sexo } & \multicolumn{2}{|c|}{ Severidad DH } & \multirow[t]{2}{*}{ Total } \\
\hline & & sin shock & con shock & \\
\hline \multirow[t]{2}{*}{$0-4$} & $\mathrm{M}$ & 2 & 1 & 3 \\
\hline & $\mathrm{F}$ & 1 & 1 & 2 \\
\hline \multirow{2}{*}{$5-14$} & $\mathrm{M}$ & 2 & 1 & 3 \\
\hline & $F$ & 4 & 2 & 6 \\
\hline \multirow[t]{2}{*}{$15-44$} & $M$ & 10 & 2 & 12 \\
\hline & $\mathrm{F}$ & 4 & 2 & 6 \\
\hline \multirow[t]{2}{*}{$>45$} & $M$ & 3 & 0 & 3 \\
\hline & $\mathrm{F}$ & 0 & 0 & 0 \\
\hline \multirow[t]{3}{*}{ Total } & $M$ & 17 & 4 & 21 \\
\hline & $\mathrm{F}$ & 9 & 5 & 14 \\
\hline & \multicolumn{4}{|c|}{$p>0.1$} \\
\hline
\end{tabular}

\section{DISCUSION}

Con el comportamiento del dengue en estos seis meses analizados, podemos hacer una proyección de lo que sería una epidemia con altas tasas de ataque.

Si tuviéramos 700.000 casos de dengue, como en otras ocasiones, alrededor de 140.000 presentarían alguna manifestación hemorrágica y al menos 40.000 requerirían hospitalización.

Con una letalidad del $3 \%$, el número de muertes estimadas sería de 1.200 aproximadamente.

Entre las regiones con mayor riesgo para dengue tenemos algunas con gran proporción de población dispersa (Arauca y Casanare), lo que nos muestra el proceso de colonización del área rural por parte del Aedes aegypti; este hecho le permitirá a este vector, transformarse en vehículo propagador de fiebre amarilla desde las zonas selváticas cercanas. Urge en estas áreas un reconocimiento de los hábitos y ecología de A. aegypti, siendo recomendable la vacunación AntiAmarílica.

El sector más afectado de la población, fue aquel en edad económicamente activa (15-44 años), con la consiguiente repercusión por días laborales perdidos.

El porcentaje de casos con DH del total de casos con dengue $(6 \%)$, es similar al reportado por Cuba (7\%). Este hecho señala la similitud en la virulencia de la enfermedad para ambos países (4).

El sexo femenino mostró una mayor positividad global para dengue. 
Los menores de 5 años tuvieron un porcentaje de positividad para DH mayor que el de otros grupos de edad, lo que está de acuerdo con lo reportado por otros países.

El pico de transmisión se alcanzó alrededor de la sexta semana epidemiológica de 1990. Probablemente las medidas de control del vector, ordenadas por el Comité Nacional de Lucha Contra el Dengue, hayan cortado la transmisión de la enfermedad.

Es posible también, que por la Vigilancia Activa desarrollada, se detectaron los casos de DH no diagnosticados en años anteriores. Es de señalar, que en países asiáticos el $\mathrm{DH}$ se presentó inicialmente en forma de brotes atípicos, para terminar seleccionando grupos de riesgo y convirtiéndose en un grave problema de Salud Pública (4).

Es urgente continuar la lucha contra el vector de esta enfemedad, para la cual tenemos todos los factores de riesgo que podrían convertirla en una grave amenaza para nuestra población. La búsqueda de otros vectores en nuestro país como el Aedes albopictus; también debería formar parte del plan de lucha contra la enfermedad.

El promedio de títulos $\mathrm{HI}$ en los primeros díez días de enfermedad, es significativo entre los pacientes con dengue, comparando con el hallado en personas que no seroconvirtieron en la segunda muestra de acuerdo a ésto, es posible que un título de anticuerpos mayor de 1:320 para cualquier serotipo, en los primeros diez días de enfermedad, sea muy sugestivo de contacto reciente con un flavivirus.

No es descabellado pensar que la mayoría de casos de $\mathrm{DH}$ ocurridos en el país durante este período, fueron causados por los serotipos 1 y 4 , ya que éstos fueron los más frecuentemente aislados durante el primer semestre de 1990.

La tasa de letalidad para DH en Colombia fue del $3 \%$ semejante a la reportada por países asiáticos y latinos.

\section{CONCLUSIONES}

Es de vital importancia continuar la Vigilancia e Investigación Epidemiológica Intensificada, hallar respuesta a los interrogantes planteados por la comunidad científica, tales como: factores de riesgo para la aparición del síndrome, respuestas del vector a medidas de control, hábitos del $A$. aegypti en zonas rurales etc., con el fin de tomar las medidas de control necesarias en forma oportuna y eficiente. Si no mejoramos nuestros sistemas de Vigilancia sobre esta enfermedad y los instituimos permanentemente, podríamos estar abocados a una epidemia de DH/SS de grandes proporciones, con las consiguientes repercusiones negativas en todos los aspectos de la vida nacional.

\section{SUMMARY}

We show here the results obtained of the Active Surviellance of the National System of Health on Classic Dengue (CD), Classic Dengue with Hemorrhagic Feactures (CD/HF) Hemorrhagic Dengue (HD) and Hemnorrhagic Dengue with Socho Syndrome (HD/ SS), during first semester in 1990.

From the total 3.098 samples revieved, $568 \mathrm{CD}$ (18\%) were confirmed, 121 Showed Hemorrhagic Feactures $(21 \%)$ and 35 were considered as HD $(6 \%)$, accoding to the OMS requirements including the last 35 cases, 9 of them presening shock $(26 \%)$, on of which was fatal $(3 \%)$.

The reions showing more $\mathrm{CD}$ incidence were: Arauca and Casanare. For HD, the regions with high incidence in Colombia were Arauca, Magdalena and Norte de Santander.

\section{BIBLIOGRAFIA}

1. INS-MINSALUD. Vigilancia y Control del Dengue Clásico y Dengue Hemorrágico 1990.

2. DANE. "Censo Poblacional, 1985".

3. White C. "Statiscal Methods in Serum Survey" en Paul J.: "Serological Epidemiology" New York Academic Press. 1972. Capítulo 3.

4. Gubeler D. "Surveillance dor Dengue Fever and Dengue hemorragic Fever" Bull, Paho 23 (4) 1989.

5. OMS "Fiebre Hemorrágica Dengue, Diagnóstico, Tratamiento y Prevención". Ginebra. 1986.

6. Daniels W. "Estadística Aplicada a las Ciencias Sociales" McGraw-Gill 1980

7. Bravo J, Guzmán M. et al. "Why Dengue Hemorragic Fever in Cuba I. Individual Rish Factors for DHF/SSD" Tr. Roy Soc. 81. 1987: p 143-146. 1987.

8. Boshell J, Groot H. et al. "Dengue en Colombia" Biomédica Vol. 6 Nos. 3 y 41986.

9. Kouri G, Guzmán M. "Criterios utilizados durante la epidemia de dengue hemorrágico para definir casos positivos y las respuesta primarias y secundarias en la prueba $\mathrm{HI}^{\prime \prime}$. Cuba 1981. Rev Cub Med Trop 35. 13-19 enero-abril 1983. 